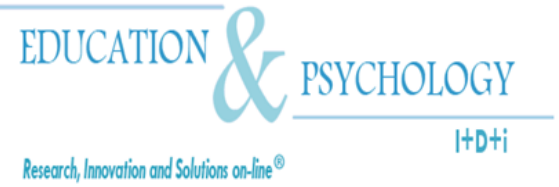

\title{
Habilidades cognitivas, conducta y potencial de aprendizaje en preescolares con síndrome Down
}

\section{Dolores Calero García ${ }^{1}$, M. Auxiliadora Robles Bello², M. Belén García Martín ${ }^{1}$ \\ ${ }^{1}$ Facultad de Psicología, Departamento de Personalidad, Evaluación y Trata- miento Psicológico. Universidad de Granada \\ ${ }^{2}$ Facultad de Humanidades, Departamento de Psicología. Universidad de Jaén}

\section{España}

Correspondencia: M. Dolores Calero García. Facultad de Psicología. Universidad de Granada. Campus La Cartuja. España. E-mail: mcalero@ugr.es 


\section{Resumen}

Introducción. Hace algunos años que se señala la necesidad de buscar un sistema de evaluación de los niños con déficits intelectuales que señales sus peculiaridades y nos informe sobre las variables que pueden favorecer su aprendizaje. Este objetivo es más interesante si cabe si puede aplicarse en niños que están en proceso de desarrollo. Desde hace 30 años se vienen desarrollando técnicas de evaluación del potencial de aprendizaje como una alternativa a la evaluación tradicional útil para tal fin. En este trabajo nos proponemos analizar y señalar las diferencias en habilidades cognitivas, actitudes y potencial de aprendizaje entre preescolares Down y preescolares de inteligencia media.

Método. Han participado 32 niños entre 4-6 años de cada grupo, evaluados con la EHPAP (versión española de la Application Cognitive Funtion Scale) instrumento que evalúa habilidades y potencial de aprendizaje según un formato de test-entrenamiento-test y se acompaña de una escala de observación de conducta y del Test de inteligencia K-bit.

Resultados. Los resultados muestran las diferencias en habilidades cognitivas, actitudes hacia el aprendizaje y potencial entre ambos grupos. Así mismo, ponen de manifiesto la utilidad de esta metodología para programar la intervención en niños con déficits.

Palabras Clave: habilidades cognitivas, potencial de aprendizaje; síndrome Down, modificabilidad cognitiva, preescolares

Recibido: 23/09/09 Aceptación Inicial: 23/09/09 Aceptación Definitiva: 12/02/10 


\title{
Cognitive skills, behavior and learning Potential of preschool children with Down syndrome
}

\begin{abstract}
Introduction. Since some years ago, it has been pointed out the necessity to search for an assessment method for children with deficits that indicate their special features and inform about the variables which can help their learning process. This objective is even more interesting if it can be applied in children which are in the development process. Since 30 years ago, learning potential techniques have been developed as a useful alternative to traditional assessment to achieve this goal. In this study, there will be analyzed and pointed out the differences in abilities, attitudes and learning potential between preschooler with Down's Syndrome and preschooler with normal intelligence.

Method. There have been participated 32 children in each group, evaluated with the Spanish version og the ACFS (Lidz and Jepsen, 2003) as an instrument to assess abilities and learning potential with a test-training-test format and an observational behaviour scale and the k-bit intelligence test of Kaufman and Kaufman (1996).

Results. The results show differences in cognitive abilities, attitudes and learning potential between the two groups. Also, the results show the usefulness of this methodology in order to programme the intervention with children with disabilities
\end{abstract}

Keywords: cognitive abilities, learning potential, Down syndrome, cognitive modifiability, preschools.

Received: 09/23/09 Initial Acceptance: 09/23/09 Definitive Acceptance: 02/12/10 


\section{Introducción}

\section{Perfil cognitivo en Síndrome Down}

Desde hace algunos años, investigadores y educadores que trabajan con niños con Síndrome Down (S.D.) señalan la necesidad y la importancia de ofrecer, a este grupo de personas, una educación de calidad que tenga en cuenta sus peculiaridades para obtener el máximo potencial. Como Fidler y Nadel (2007) señalan, la investigación sobre el fenotipo asociado al S.D. ha ayudado a comprender su perfil cognitivo pero se requiere más información sobre su modo de aprender y sobre las variables que favorecen y/o dificultan este proceso para diseñar procedimientos de intervención efectivos.

Un discreto número de trabajos de investigación han dirigido a analizar las diferencias en ejecución de los niños Down respecto de niños sin déficits o con otro tipo de déficits intelectuales encontrando una significativa relación entre su falta de estrategias de exploración y su poca flexibilidad en la infancia temprana con una peor coordinación oculo-manual (Arnaiz, 1991); también se ha señalado que, debido a sus alteraciones cerebrales, posee déficits atencionales, su velocidad para analizar y procesar información es menor y su habilidad de resolución de problemas, así como su memoria a largo plazo, menos precisas (Chapman y Hesketh, 2000; Flórez, 1999). En concreto, se destaca su tendencia a la distracción y falta de concentración (Florez, 1999; Vived, 2004), su peor memoria explícita y memoria auditiva a corto plazo (Carselimo, Marotta y Vicari,1997; Pérez Sánchez, Beltrán y Sánchez, 2006), sus problemas con la memoria declarativa (episódica y semántica) (Nadel, 2000), sus dificultades fonológicas y de procesamiento verbal (Gunn y Jarrold, 2004; Laws y Bishop, 2003) y su lentitud en la adquisición de habilidades pragmáticas (Rondal, 2000). Tambien se ha comprobado que tanto la capacidad de discriminación auditiva como la visual están más afectadas en estos niños que en otros tipos de déficits intelectuales con niveles equivalentes (Kennedy y Sheridan, 1973).

Otros aspectos de interés para la programación de intervenciones como son los emocionales también han sido estudiados. Así, se ha señalado como características significativas su impulsividad,- tendencia a responder sin planificación-, su baja tolerancia a la frustración, o escasa persistencia en la realización de una tarea, (Glen y Cunnigham, 2002; Vived, 2004), su baja motivación intrínseca (Willians, Wishart, Pitcairn y Willis, 2005) y su necesidad de 
regulación externa para realizar una tarea (Glenn y Cunnighan, 2002), a pesar de que se ponga de relieve que suelen ser individuos sociables e interesados en el contacto social (Ruiz, 2001).

A pesar de los déficits apuntados, algunos autores señalan que existen evidencias de su posibilidad de modificación cognitiva, esto es de la mejora de su ejecución intelectual, sobre todo cuando en los programas de entrenamiento se tienen en cuenta los aspectos motivacionales. Así se indica que poseen cierta capacidad de aprendizaje pero con inestabilidad en la adquisición y adquisición mas lenta que en los niños no-Down (Candel, 2005; Fidler y Nadel, 2007). En este sentido varios autores apuntan los beneficios del enriquecimiento del ambiente (interacciones madre-hijos, etc...) como factor de modificación y cambio positivo en este grupo (Pino, 2000; Taubman, Vrierley, Wishner, Baker, McEachin, y Leaf, 2001).

Aunque desde hace tiempo que se reconoce la importancia de estos otros aspectos no cognitivos en el aprendizaje, hasta el momento no se han publicado trabajos que examinen conjuntamente las habilidades, las actitudes y la capacidad de aprendizaje de niños Down, probablemente debido a la escasez de métodos de evaluación, diferentes de los tests de inteligencia, aplicables a este grupo.

En relación con este panorama, algunos autores han señalado la necesidad de buscar un sistema de evaluación alternativo a los tests tradicionales de inteligencia que informe de las peculiaridades de cada grupo deficitario, (y en concreto de los niños Down) y ayude a la planificación de intervenciones útiles; una evaluación que refleje objetivos curriculares específicos para promover una mayor y estrecha relación entre la evaluación y la instrucción (Elliott y Fuchs, 1997; Fuchs y Fuchs, 1996).

Una de las aproximaciones que con más fuerza se ha presentado como complemento o alternativa a los tests de inteligencia ha sido la denominada evaluación dinámica o evaluación del potencial de aprendizaje, aproximación que, basándose en los conceptos de ZDP ${ }^{l}$ (Zona de Desarrollo Próximo) de Vigotsky y de modificabilidad cognitiva ${ }^{2}$ de Feuerstein, se dirige

\footnotetext{
${ }^{1}$ ZDP: Distancia entre el nivel ral de desarrollo, determinado por la capacidad de resolver independientemente un problema, y el nivel de esarrollo potencial, determinado a traves de la resolución de un problema bajo la guia de un adulto o en colaboración con un compañero más capaz. (Vigotsky, 1978 pg. 133)

${ }^{2}$ Modificabilidad cognitiva se define como una transformación en la estructura del intelecto del individuo que refleja un cambio en el curso esperado de su desarrollo (Feuerstein, 1997.Pg.28). Hace referencia al potencial del sujeto para adaptarse al cambios en factores externos e internalizar los elementos resultantes (Feuerstin et al, 2002, pg. 130) 
a evaluar la posibilidad de aprendizaje de una persona que inicialmente posee una ejecución baja en una tarea; para ello, mediante un diseño pretest- intervención/mediación -postest sobre una tarea similar a las utilizadas por los tests de inteligencia tradicionales, permite valorar la capacidad de aprendizaje de un individuo y establecer algunas variables relacionadas con la tarea, el contexto y/o con el propio sujeto que pueden facilitar dicha modificabilidad. Esta metodología ha mostrado su utilidad para poblaciones deficitarias (Budoff, 1969; Calero, 2004; Campione y Brown, 1987; Carlson y Wiedl, 2000; Fernández-Ballesteros y Calero, 2000; Feuerstein, Rand y Hoffman,1979; Feuerstein, Feuerstein, Falik y Rand, 2002; HesselsSachltter, 2002; Lauchlan y Elliott, 2001; Lidz y Thomas, 1987; Tzuriel y Klein, 1985), pues diferentes autores han puesto de manifiesto la modificabilidad cognitiva (el aumento significativo de puntuaciones tras la fase de intervención) de niños con déficits intelectuales, de aprendizaje y/o de lenguaje y son muchos los que ven en este tipo de evaluación un importante instrumento de apoyo psicopedagógico y una oportunidad para mejorar los procesos de enseñanza-aprendizaje dado que la fase de mediación promueve capacidades de autorregulación del aprendizaje y la adquisición de estrategias metacognitivas (Fuchs, Fuchs y Hamlett, 1994; Lidz y Gindis, 2003; Samir y Lazerovitz, 2007; Sternberg y Grigorenko, 2003; Rodríguez, 2005). Su objetivo no es sólo medir la ejecución de los sujetos, sino su posibilidad de aprendizaje, su fin no es el pronóstico académico, entendido del modo tradicional, sino la estimación de la posibilidad de aprovechamiento de diferentes programas de entrenamiento cognitivo. (Calero, 2004; Haywood y Lidz, 2007).

De hecho existe gran cantidad de trabajos que muestran como niños con déficits intelectuales mejoran significativamente su ejecución en tests tradicionales de inteligencia o rendimiento mediante estos procedimientos (Swanson y Lussier, 2001) y como ésta modificabilidad predice su posibilidad de mejora en diferentes habilidades, - tales como memoria visual y auditiva, clasificación, seriación, lectura, memoria episódica y semántica, etc...-, cuando se planifican intervenciones intensivas, esto es, puede servir para establecer la respuesta a la intervención (Calero y Márquez, 1998; Carfrey, Fuchs y Fuchs, 2008).

Aunque la mayoría de las publicaciones no distingue entre S.D. y otros grupos con déficits intelectuales podemos señalar algunos trabajos realizados con esta metodología en niños Down.En concreto, Molina y Arraiz (1993), realizan un estudio con 180 sujetos, con edades medias en torno a los 10 años, de los que 30 presentaban síndrome de Down (SD), 60 poseían discapacidad intelectual ligera y 30 inteligencia normal establecida según un test 
estándar de inteligencia. En sus resultados encontraron que las puntuaciones pretest eran significativamente más bajas en el grupo SD que en el de discapacidad ligera pero las diferencias disminuyeron al administrar entrenamiento, llegando a desaparecer la significación en tareas de planificación. Ortega (2002) con 8 niños con síndrome de Down, de una media de 7 años de edad, encontró que los niños del grupo experimental con entrenamiento mediante ordenador en tareas de cálculo obtuvieron una significativa mejor puntuación en el postest. Robles y Calero (2003) con una muestra de niños SD de 12 años de edad media encontraron que tras recibir tratamiento, el grupo presentaba una significativa mejor puntuación en una tarea de lectura.

Si interesante es establecer la modificabilidad en niños con déficits más aún lo es si estos son de edad preescolar pues, en estos niños, las habilidades evaluadas están en proceso de desarrollo y las posibilidades potenciales de una buena intervención son mayores y más importantes. Una de las líneas fundamentales de investigación centrada en la aplicación de evaluación dinámica a población preescolar ha sido la desarrollada por Carol Lidz. Esta autora que comenzó aplicando el esquema de Feuerstein a educación preescolar, finalmente ha desarrollado una escala de evaluación dinámica (Lidz, 1991, 2002; Lidz y Jepsen, 2003). Lo diferente y específico de su aproximación está en que se interesa por realizar una adaptación de la evaluación dinámica al contexto educativo, por ello las tareas que utiliza para la evaluación se toman directamente del currículum académico en el que el niño/a puede mostrar dificultades de aprendizaje. En 2000/03, Lidz y Jepsen proponen la Application Cognitive Funcion Scale (ACFS), como una escala diseñada específicamente para niños de edades comprendidas entre 3 y 5 años de edad que evalúa, -mediante un formato pretest-entrenamiento/ mediaciónpostest-, la aplicación de estrategias de aprendizaje a tareas típicas del currículo y que además incluye una escala de observación y estimación de conductas mediante la cual el evaluador califica siete parámetros de la conducta del niño durante las fases de pretest y mediación de los seis subtests que componen la escala.

La fiabilidad y validez de la ACFS ha sido ampliamente demostrada con diferentes tipos de poblaciones procedentes de distintos países con gran variedad de niveles socioeconómicos y étnicos (Aranov, 1999; Bensousan, 2002; Levy, 1999; Lidz y Van der Aalsvoort, 2005; Malowitsky, 2001; Shurin, 1998). Con este instrumento se ha comprobado la modificabilidad en niños con trastornos en el desarrollo (Brooks, 1997; Shurin, 1998) con necesidades educativas especiales (Takit, 2000), sordos (Lidz, 2004) y con retraso en el de- 
sarrollo (McDonals, 2006 citado en Haywood y Lidz, 2007), en estos grupos se han encontrado perfiles diferentes de ejecución respecto de los párvulos sin déficits y además algunos de estos trabajos ponen de manifiesto las relaciones entre la baja ejecución y las puntuaciones en la conducta observada durante el proceso de aprendizaje (Aranov, 1999; McDonald, 2006; Shurin, 1998),

Respecto a los trabajos realizados con párvulos SD utilizando un formato dinámico hasta el momento cabe reseñar el trabajo de Nihlom (1999) que utilizan el concepto de ZDP de Vygotsky sobre una tarea de reproducción de un dibujo. En este estudio trabajan con 8 niños Down y 8 párvulos sin déficits para establecer diferencias cualitativas y cuantitativas en su proceso de aprendizaje y comprueba que los Down, aunque se benefician de la mediación, necesitan mayor numero de ensayos para llegar al mismo nivel de eficiencia que los normales y que, aspectos como las instrucciones y/o los estímulos que componen la tarea, influyen en su aprendizaje y les lleva a cometer errores diferentes a los niños del grupo de inteligencia media. En otro trabajo Alony y Kozulin, (2007) se centran en la evaluación dinámica del Vocabulario receptivo utilizando el Peabody Picture Vocabulary Tests (Dunn y Dunn, 1981) con un tipo de mediación focalizada que sigue los principios marcados por Feuerstein y colaboradores. Sus resultados muestran, tras la mediación, una mejora significativa en vocabulario, lo que, a juicio de sus autores, supone que una habilidad cristalizada (vocabulario receptivo) es modificable, en niños Down, mediante mediación focalizada.

En definitiva, si aceptamos que los niños Down presentan perfiles de ejecución diferentes a los niños con otros tipos déficits intelectuales y a los niños sin déficits, si estamos de acuerdo que la conducta que manifiesta el niño durante el proceso de aprendizaje puede tener una importante relación con su ejecución final; y si además mantenemos el convencimiento que los niños SD poseen potencial de aprendizaje, esto es pueden mejorar significativamente su ejecución en una tarea tras un entrenamiento (mediación) sistemático de corta duración, un objetivo relevante, -que hasta el momento no se ha acometido-, de cara al diseño de intervenciones eficaces, es determinar los perfiles diferenciales en habilidad, conductas y potencial de aprendizaje entre niños Down y niños sin déficits y este es el objetivo de la investigación que se presenta. Para atender al mismo hemos utilizado la EHPAP: Evaluación de habilidades y potencial de aprendizaje para preescolares, Versión española de la ACFS, de Lidz y Jepsen. 
Este propósito se puede operacionalizar en una serie de objetivos específicos tales como: 1) Comprobar las diferencias en la ejecución de partida (puntuación prestest) entre los niños con Síndrome de Down, y preescolares sin déficits. 2) Demostrar la efectividad de la fase de mediación (o intervención sobre la tarea) que forma parte de esta prueba, en los dos grupos de sujetos y 3) Comprobar si existe un determinado perfil de comportamiento, en función del grupo que se trate.

\section{Método}

\section{Participantes}

La muestra ha estado compuesta por 64 niños de edades entre 3 y 6 años, todos ellos asistentes a diferentes colegios públicos de la provincia de Jaén en España. Está dividida en dos grupos, el primero de 32 niños con Síndrome de Down de 3- 6 años de edad $(M=4.46$; d.t.= 1.13), integrado por 7 niñas y 25 niños; C.I medio total según el K-bit de de Kaufman y Kaufman (1994) de 53.31 (d.t. 14.97). El segundo grupo de 32 niños no -Down (desde este momento identificados como preescolares) de 3- 6 años de edad $(\mathrm{M}=4.59$; d.t.=0.79), integrado por 11 niñas y 21 niños. C.I. total según K-bit de media 90.97; d.t.=13.91.

\section{Instrumentos}

Test breve de inteligencia de Kaufman (K-BIT) (Kaufman y Kaufman, 1994). Es un test de "screening" que permite llegar a una rápida apreciación de la inteligencia general del niño mediante dos subtests: vocabulario y matrices. Con el primero se evalúan habilidades verbales, relacionadas con el aprendizaje escolar y con el segundo la capacidad para resolver problemas de razonamiento. Suministra un C.I. verbal, un C.I. no verbal y un C.I. compuesto que resume el rendimiento total en el test. Los estudios sobre validez y fiabilidad demuestran que sus coeficientes de fiabilidad varían según el rango de edad, pero en ningún caso baja del 0,76; además el C.I. compuesto muestra una correlación media de 0,63 con la suma de puntuaciones de procesamiento mental del K-ABC y de 0,75 con la del subtest de conocimientos del mismo test. También, el C.I. compuesto del K-BIT tiene una correlación de 0,80 con el C.I. global del WISC-R y de 0,75 con el WAIS-R. Estas correlaciones apoyan la validez de constructo de los C.I. compuestos del K-BIT. 
EHPAP: Evaluación de habilidades y Potencial de aprendizaje para preescolares (Calero, Robles, Márquez y de la Osa, 2009) Versión española de la Escala de Aplicación de Funciones Cognitivas: ACFS de Lidz y Jepsen (2000/03). La EHPAP es una escala de habilidades administrada con un formato de evaluación de pretest-intervención/mediación-postest. El pre y el post de cada subescala son similares o con pequeñas variaciones de la misma tarea, con unas instrucciones estandarizadas para su administración, ya que con ellas se trata de evaluar la ejecución real del niño, sin ayuda, en tareas próximas al curriculum educativo. Durante la fase de intervención o mediación, se introduce un dialogo mediado entre aprendiz y mediador con el que se trata de instruir (con señales verbales, modelado y feedback sobre la ejecución) en la realización de una tarea paralela, pero no igual, a la que se utiliza en las fases pre y post. Esta fase se dirige a generar una estrategia de solución de las diferentes tareas suministrándole ayudas graduadas. Posteriormente se evalua con el posttest. La EHPAP suministra una puntuación que indica el grado en que los niños han dominado cada una de las tareas (pretest), y otras indicativas del nivel de receptividad del niño a la intervención (postest) y su potencial de aprendizaje (puntuación de ganancia y/o transferencia: diferencia post-pre). Las habilidades cognitivas evaluadas por los seis subtests son las siguientes:

1. Clasificación. la tarea consiste en que el niño haga grupos con bloques de madera según un criterio (forma, color, tamaño)

2. Memoria Auditiva. En este se lee al niño un pequeño cuento, que debe escuchar para contarlo después con una secuencia temporal correcta.

3. Memoria Visual. En esta actividad, se le pide al niño que diga el nombre de los objetos que hay impresos en ocho láminas y una vez retirados se pide su recuerdo.

4. Secuencia de patrones. En esta tarea se le presentan al niño diferentes series de formas geométricas que tiene que completar con la pieza correcta, elegida de entre varias posibles.

5. Toma de Perspectiva. En esta prueba, se le pide al niño que asuma la perspectiva del evaluador y que utilice todas aquellas indicaciones que considere necesarias para enseñarle a realizar un dibujo.

6. Planificación verbal. Este requiere que el niño diga la secuencia conductual temporal necesaria para realizar una tarea concreta.

Como parte de la EHPAP, estos seis subtests se complementan durante su aplicación con la Escala de Observación Conductual (EOS), que incluye diversas dimensiones relativas al comportamiento del niño que son evaluadas mediante observación en cada uno de los pre- 
tests y las fases de mediación de los 6 subtests de ejecución. Las áreas evaluadas por la EOS son:

- Autorregulación: Hace referencia al grado en que el niño controla la atención e inhibe la respuesta impulsiva.

- Persistencia: hace referencia a si el niño trabaja hasta la conclusión de la tarea con independencia del éxito obtenido en la resolución.

- Tolerancia a la Frustración: Se relaciona con la capacidad que el niño tiene para continuar trabajando a pesar de la experiencia de fracaso.

- Flexibilidad: Hace referencia a la búsqueda de soluciones alternativas o autocorrecciones que el niño realiza mientras resuelve las tareas.

- Motivación: Está relacionada con las demostraciones de interés y reacciones de entusiasmo que muestra el niño hacia los materiales y hacia la tarea.

- Interactividad: Hace referencia a su apertura a la interacción con el mediador.

- Receptividad: Hace referencia a si atiende a las instrucciones o demostración del mediador.

- Como ya hemos comentado, tanto la EHPAP como la escala de observación conductual que incorpora, han mostrado su fiabilidad (Shurin, 1998; Levy, 1999) y validez de constructo (Malowitsky, 2001) y concurrente (Takit, 2000) y comprobado su utilidad con diferentes poblaciones.

\section{Procedimiento}

En primer lugar se llevó a cabo la traducción y adaptación al castellano de la prueba original. Posteriormente a través de la Asociación Down de Jaén se contactó con los padres de los niños que tenían entre 3 y 5 años. Una vez fijado el grupo de niños Down se paso a seleccionar un grupo similar de preescolares sin déficits que no presentaran ningún otro problema de conducta. Una vez obtenido el consentimiento de los padres se procedió a la evaluación con los dos instrumentos (el K-Bit yla EHPAP). Todas las sesiones se realizaron de forma individualizada con una duración aproximada de dos horas por niño con descansos entre subtests en aquellos niños que los requerían. 


\section{Diseño y Análisis Estadísticos}

El diseño seguido en esta investigación ha sido cuasiexperimental con grupos 2 equivalentes establecidos según el diagnóstico: Síndrome de Down, y Preescolares. Los análisis estadísticos realizados han consistido en comparaciones t de Student de diferencia de medias, ANOVAs de medidas repetidas, cálculo de tamaño de efecto y un análisis discriminante por pasos todos ellos realizados utilizando el paquete estadístico SPSS 15.0.

\section{Resultados}

En primer lugar se comprobó que no existían diferencias significativa entre los dos grupos de sujetos que componen la muestra en relación con la edad $(\mathrm{t}(62)=.510 ; \mathrm{p}=.612)$; los grupos, no obstante no tiene la misma proporción de varones/mujeres debido a que no se se realizó selección alguna de los niños Down, sino que participaron todos los que pertenecían en ese momento a la asociación y tenían la edad establecida. Posteriormente pasamos a establecer el perfil diferencial de ejecución en los distintos subtests de la EHPAP entre niños Down y preescolares esto es, la puntuación obtenida en la fase de pretest. Como se puede observar en la figura 1, y ponen de manifiesto los ANOVAS realizados, aparece una significativa mayor ejecución a favor de los preescolares, en todos los subtests que componen la escala, Clasificación $(\mathrm{F}(1 / 63)=20.33 \mathrm{p}<.0001)$, Memoria Auditiva $(\mathrm{F}(1 / 63)=43.75 \mathrm{p}<.0001)$, Memoria Visual $(F(1 / 63)=174,92 p<0,0001)$, Ejecución de Patrones $(F(1 / 63)=34.20 ; p<.0001)$ y Toma de Perspectiva $(\mathrm{F}(1 / 63)=49.65 ; \mathrm{p}<.0001)$ y Planificación Verbal $(\mathrm{F}(1 / 63)=10.00$ $\mathrm{p}<.002$ ). Igualmente en la puntuación pretest total de la EHPAP (suma de las puntuaciones obtenidas en todos los subtests que componen la escala en la situación pretest) aparecen diferencias entre grupos que resultan estadísticamente muy significativas $(F \quad(1 / 63)=83.34$; $\mathrm{p}<.0001)$.

Respecto del segundo objetivo que perseguía establecer la efectividad de la fase de mediación sobre las habilidades evaluadas por las EHPAP en los dos grupos; como se refleja en la tabla 1 se comprueba que efectivamente existen diferencias significativas entre las puntuaciones pretest y postest de los diferentes subtests de la EHPAP para los dos grupos que componen la muestra. 
Comparación de las medias de los grupos PREESCOLAR-SÍNDROME DE DOWN en la situación PRETEST de la ACFS.

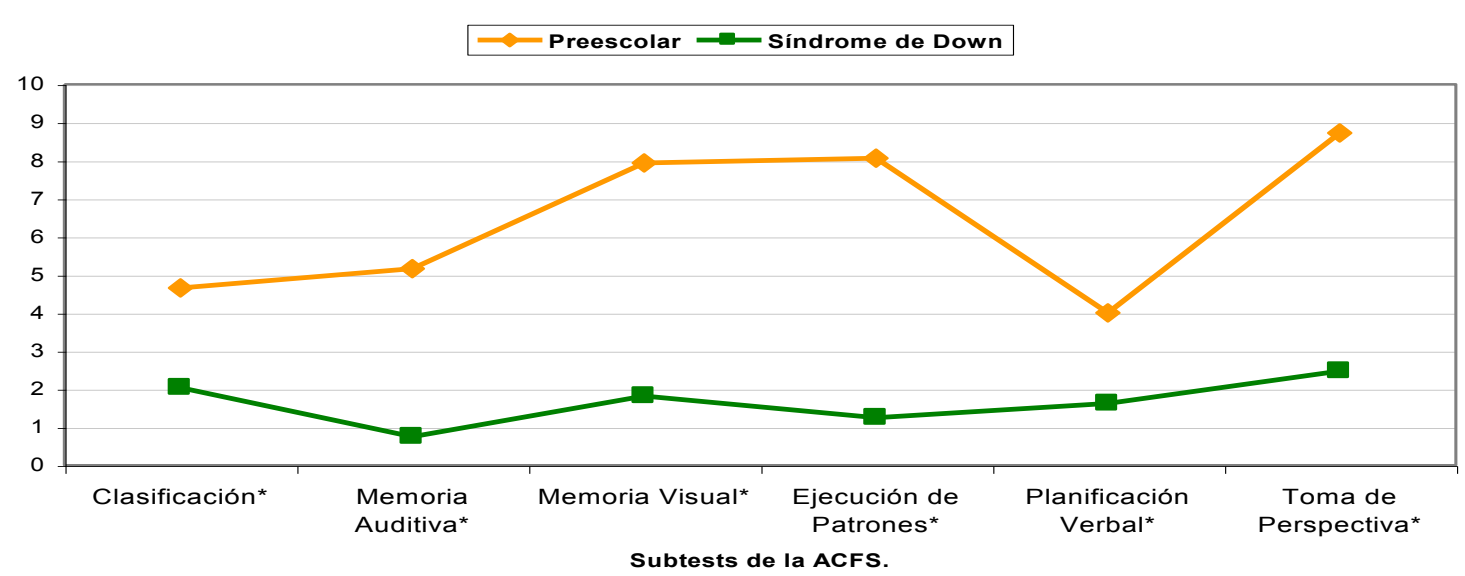

Figura 1. Comparación de las medias de los grupos Preescolar y Síndrome de Down en la situación pretest de la ACFS para todos los subtests. $(*=\mathrm{p}<.05 ; * *=\mathrm{p}<.001)$

Tabla 1. Puntuaciones pre, post y ganancia de los grupos Down y preescolares en cada subtests de la ACFS. Se incluye el tamaño de efecto de la diferencia pre-post (d de Cohen).

\begin{tabular}{lcccccccc}
\hline Subtest & \multicolumn{3}{c}{ DOWN } & \multicolumn{4}{c}{ PREESCOLARES } \\
\hline & PRE & POST & G & d & PRE & POST & G & d \\
\hline Clasificación & 2.0313 & 3.0938 & 1.2118 & $.6867^{*}$ & 4.6562 & 7.2188 & 2.5625 & $1.7556^{* *}$ \\
& 2.32 & 2.33 & 1.10 & & 2.33 & 1.82 & 1.60 & \\
Memoria Auditiva & 0.7500 & 1.7813 & 1.0313 & $.7362^{*}$ & 5.1562 & 7.0313 & 2.7188 & $.9584^{* *}$ \\
& 1.19 & 2.63 & 2.07 & & 3.57 & 3.94 & 2.91 & \\
Memoria Visual & 1.8125 & 3.8437 & 2.0313 & $1.2521^{* *}$ & 7.9375 & 8.4062 & 0.7188 & .3671 \\
& 2.13 & 3.00 & 1.63 & & 1.52 & 1.68 & 1.37 & \\
Ejecución de Patrones & 1.2500 & 1.9688 & 0.7188 & $.4676^{*}$ & 8.0625 & 10.437 & 2.3750 & $.9280^{* *}$ \\
& 1.97 & 2.55 & 1.17 & & 6.29 & 6.45 & 3.62 & \\
Planificación Verbal & 1.6250 & 2.6563 & 1.0313 & $.7658^{* *}$ & 4.0000 & 4.6875 & 0.6875 & $.3755^{*}$ \\
& 2.82 & 3.61 & 1.91 & & 3.17 & 3.24 & 1.40 & \\
Toma de Perspectiva & 2.4688 & 4.0313 & 1.5625 & $.6971^{*}$ & 8.7188 & 10.000 & 1.1250 & $.7220^{*}$ \\
& 4.17 & 5.73 & 3.06 & & 2.78 & 3.45 & 2.38 & \\
\hline
\end{tabular}

Así, respecto a la cuantía de las ganancias obtenidas tras la intervención, los ANOVAs realizados muestran que el grupo de preescolares, en general, mejora tras la intervención significativamente más que el grupo de niños Down; presentando diferencias en la puntuación de ganancia (post-pre) entre grupos muy significativas en Clasificación $(F(1 / 63)=20.39 ; p<.0001)$ y en el Memoria Auditiva $(F(1 / 63)=7.17 ; p<.001)$ y significativas en Ejecución de Patrones $(\mathrm{F}(1 / 63)=3.81 ; \mathrm{p}<.012)$. Sin embargo los niños Down mejoran significativamente más que los preescolares en el subtest Memoria Visual $(F(1 / 63)=8.20 ; p<.0001)$ 
y en el de Toma de Perspectiva (F (1/63)=3.94; $\mathrm{p}<.01)$ y no aparecen diferencias significativas en los resultados de la mediación entre grupos en el subtest de Planificación Verbal (F $(1 / 63)=2.00 ; p<.117)$. Por, ultimo, al analizar el tamaño del efecto, que nos ayuda a determinar la magnitud de la diferencia pre-post en cada variable, encontramos que las d de Cohen obtenidas son significativas en todos los casos para el grupo Síndrome de Down, y en todos los subtests excepto en el de Memoria Visual para el grupo Preescolar. Todo lo cual afianza aún más la hipótesis sobre la eficacia de la intervención en todos los niños independientemente de grupo de pertenencia, ya que los resultados no sólo son estadísticamente significativos sino que se confirman y son relevantes desde una perspectiva clínica. En definitiva los resultados muestran un significativo potencial de aprendizaje en ambos grupos de niños.



Figura 2. Diferencias entre pretest y mediación en la Escala de Observación de Conducta en los grupos Síndrome de Down y Preescolar.

Para comprobar el tercer objetivo que consistía en determinar el perfil diferencial de comportamiento de los niños, -evaluado mediante la Escala de Observación de Conducta -, llevamos a cabo ANOVAs entre grupos. Como puede observarse en la figura 2, que representa las puntuaciones obtenidas en cada conducta en las fases de pre y mediación para cada grupo. El grupo Down parte de un pretest significativamente mas bajo que el preescolar, y así lo ponen de manifiesto los ANOVAs intergrupos realizados para cada uno de lo componentes de conducta evaluados: Autorregulación $(\mathrm{F}(1 / 63)=45.97 ; \mathrm{p}<.0001)$, Persistencia $(F(1 / 63)=46.314 ; p<.0001)$, Tolerancia a la Frustración $(F(1 / 63)=27.34 ; p<.0001)$, Flexibilidad $(\mathrm{F}(1 / 63)=39.81 ; \mathrm{p}<.0001)$, Motivación $(\mathrm{F}(1 / 63)=22.02 ; \mathrm{p}<.0001)$ e Interacción $(F(1 / 63)=27.84 ; p<.0001)$. En la fase de mediación, se mantienen las diferencias entre grupos 
(Down /preescolares), pero mientras que los niños preescolares no sufren apenas variabilidad en sus comportamientos entre la situación pre y la de mediación, -y así se refleja en los anovas realizados siguiendo un modelo lineal general de medidas repetidas para cada uno de los componentes de conducta de la escala (Autorregulación $(F(1 / 31)=1.493 ; \mathrm{p}=.231$ ), Persistencia $(\mathrm{F}(1 / 31)=1.000 ; \mathrm{p}=.325)$, Tolerancia a la Frustración $(\mathrm{F}(1 / 31)=.592 ; \mathrm{p}=.447)$; Flexibilidad $(\mathrm{F}(1 / 31)=.0001 ; \mathrm{p}=1.000)$; Motivación $(\mathrm{F}(1 / 31)=0.752 ; \mathrm{p}=.393)$, e Interacción $(\mathrm{F}(1 / 31)=.564$; $\mathrm{p}=.458)$ ), los Down presenta diferencias significativas entre ambas situaciones pretest y mediación en todas las variables de conducta: Autorregulación $(F(1 / 31)=25.317 ; p<.0001)$, Persistencia $(F(1 / 31)=11.078 ; p<.002)$, Tolerancia a la Frustración $(F(1 / 31)=23.392 ; p<.0001)$, Flexibilidad $(\mathrm{F}(1 / 31)=6.767 ; \mathrm{p}=.014)$, Motivación $(\mathrm{F}(1 / 31)=29.268 ; \mathrm{p}=.0001)$, e Interacción $(\mathrm{F}(1 / 31)=5.446 ; \mathrm{p}=.026)$.

Por último, intentamos comprobar si se puede establecer un patrón de conducta que discrimine a los niños Down del grupo de preescolares, para ello hemos llevado a cabo un análisis discriminante por etapas tomando como variable dependiente al status (Down/ preescolares) y como independientes a la puntuación pretest obtenida en las diferentes conductas evaluadas por la EOS (Autorregulación, Persistencia, Tolerancia a la Frustración, Flexibilidad, Motivación e Interacción).

A partir de los resultados de dicho análisis se obtuvo una función discriminante cuyo autovalor fue de 3.077. El porcentaje de varianza que explica esta función es de $100 \%$. Su valor de lambda de Wilks resulta significativo ya que es igual a $.245\left(\chi^{2}(3)=85.020\right.$; $p=$ $.0001)$.

La tabla 2 muestra los resultados del análisis discriminante que nos ha servido para determinar la capacidad predictiva de cada conducta respecto del grupo (Down/ preescolares). El análisis realizado, tiene en cuenta las siguientes variables: Autorregulación, Persistencia, y Motivación. Las dos primeras en sentido positivo (con coeficientes discriminantes canónicos estandarizados de .890 y 1.476 respectivamente) y la tercera en negativo (-1.619), estas son pues las variables que contribuyen significativamente a la predicción. El paso 3 muestra unas puntuaciones Lambda de Wilks relativamente bajas $(.242, .245$ y .640). En la matriz de clasificación realizada a partir de la función basada en esas tres variables mencionadas se han clasificado correctamente el $92.2 \%$ de los casos agrupados, siendo el grupo preescolar el que 
mayor porcentaje de aciertos en el pronóstico consigue (96.9\%). La significación del ajuste de la predicción es significativa $\left(\chi^{2}(3)=55.30 ; \mathrm{p}=.001\right)$.

Tabla 2. Análisis discriminante por pasos.

\begin{tabular}{ccccccc}
\hline PASO & $\begin{array}{c}\text { VARIABLE } \\
\text { INTRODUCIDA }\end{array}$ & TOLERANCIA & $\begin{array}{c}\text { F PARA } \\
\text { SALIR }\end{array}$ & $\begin{array}{c}\text { WILKS } \\
\text { LAMBD }\end{array}$ & F(d.f.) & P \\
\hline $\mathbf{1}$ & Persistencia & 1000 & 124.99 & & $124.99(1 / 62)$ & .0001 \\
$\mathbf{2}$ & Persistencia & .150 & 58.065 & .515 & $84.99(2 / 61)$ & .0001 \\
& Motivación & .150 & 15.586 & .332 & & \\
$\mathbf{3}$ & Persistencia & .104 & 12.367 & .296 & $61.524(3 / 60)$ & .0001 \\
& Motivación & .128 & 20.373 & .329 & & \\
& Autoregulación & .119 & 4.597 & .264 & & \\
\hline
\end{tabular}

En definitiva podemos decir que se ha comprobado que tres de la variables de la Escala de Observación de Conducta, en concreto, Persistencia, Flexibilidad y Motivación, discriminan significativamente los grupos establecidos y predicen la pertenencia de un sujeto a un grupo con una alta probabilidad de acierto, mayor cuando se trata de un sujeto sin Síndrome de Down.

Tabla 3. Resultado de la clasificación realizada con las variables extraídas en el análisis discriminante por pasos. Chi cuadrado $(3)=55.30 ; p>0.001$

\begin{tabular}{ccccc}
\hline MUESTRA & \multicolumn{3}{c}{ Grupo de pertenencia pronosticado } & Total \\
\hline \multicolumn{4}{c}{ Down } & Preescolares \\
Down & $(\%)$ & $28(87.5)$ & $4(12.5)$ & 32 \\
& & & $(100.0)$ \\
Preescolares & $(\%)$ & $1(3.1)$ & $31(96.9)$ & 32 \\
& & & $(100.0)$ \\
\hline
\end{tabular}

\section{Discusión y conclusiones}

Esta investigación tenía un objetivo general que consistía en comparar, mediante la escala EHPAP, las diferencias en habilidades cognitivas, conductas hacia el aprendizaje y potencial de aprendizaje entre niños preescolares normales y con Síndrome de Down.

Con respecto al primer objetivo hemos encontrado que efectivamente existen diferencias significativas en la ejecución en diferentes tareas (esto es, en las puntuaciones pretest de todos los subtests de la EHPAP) entre los grupos de niños Síndrome de Down comparados con el grupo Preescolar de inteligencia media. Los resultados muestran que las mayores dife- 
rencias de los sujetos con Síndrome de Down respecto de los preescolares, se relacionan fundamentalmente con la modalidad auditivo-verbal, tanto en memoria como en procesamiento (planificación verbal), aspectos estos que se relacionan con los déficits señalados por diferentes autores para esta población (Buckley y Perera, 2005; Buckley y Sacks, 2002; Molina y Arraiz, 1993).

Respecto a la segunda cuestión planteada, esto es la posibilidad de mejora de la ejecución tras una fase de mediación, encontramos que ambos grupos, preescolares y Down, mejoran significativamente su ejecución en el postest de la EHPAP, en prácticamente todas las habilidades evaluadas; esto es, que ambos grupos presentan un potencial de aprendizaje clínicamente significativo si consideramos el tamaño de efecto encontrado para cada habilidad, dato concordante con los trabajos previos realizados con esa escala en diferentes tipos de poblaciones (Bensousan, 2002; Brooks,1997; Levy,1999; Malowitzky, 2001; Shurin,1998). Más interesante aún supone el que, aunque los preescolares mejoran significativamente más en algunas habilidades (en concreto en Clasificación y Memoria Auditiva) los niños Down mejoran significativamente más en otras, en concreto, en Memoria visual, - resultado que está en consonancia con aquellos trabajos que han mostrado que este tipo de memoria es un "punto fuerte" en las personas con Síndrome de Down (Buckley, Bird, Sacks y Perera, 2005; Comes, 2006; Comes, Aznar, Contijoch, y Vives, 2001). Tambien se muestra una mejora significativa en su ejecución en toma de perspectiva, resultado que puede relacionarse con la mejor respuesta de los niños Down a la interacción social que varios autores han puesto de manifiesto (Pino, 2000; Fidler y Nadel, 2007; Taubman et al., 2001) y en planificación verbal. Siendo, este último, uno de los subtests en los que peor ejecución inicial presentaban. Estos resultados son concordantes con los resultados que sobre su modificabilidad respecto de otra habilidad como es el vocabulario expresivo, señalan Alony y Kozulin (2007).

Por último, respecto de los comportamientos evaluados por la EOS, cuando comparamos los diferentes componentes de conducta en situación de evaluación y de aprendizaje dentro de un mismo grupo encontramos que mientras que el grupo de niños preescolares presenta la misma conducta en pretest que en la fase mediación, -lo que significa que no mejora su conducta durante el entrenamiento-, en el grupo Síndrome de Down aparecen diferencias significativas entre pretest y mediación en todas las conductas evaluadas; es decir, el grupo Síndrome de Down no sólo se beneficia de la mediación en su ejecución cognitiva sino que mejora en todos los componentes de conducta evaluados por esta escala como respuesta al 
entrenamiento. Los datos recogidos ponen, por tanto, de manifiesto que, en el caso de los componentes conductuales, la mediación es beneficiosa sólo para aquellos individuos que parten de una posición más deficitaria ya que el grupo Down es el que más puntuación de ganancia experimenta en este sentido, lo cual también se observa en los trabajos de Molina (2002), Nihlom (1999) y Alony y Kozulin (2007) y está en consonancia con los trabajos que señalan la respuesta positiva de los Down a un entrenamiento basado en la interacción social (Taubman et al., 2001).

Además, encontramos que tres variables conductuales (Persistencia, Flexibilidad y Motivación) son las que discriminan significativamente y se relacionan con la ejecución diferencial del grupo Síndrome de Down frente al grupo de preescolares en la escala EHPAP. Diferentes autores señalan que los aspectos negativos de la conducta de los Down que deben ser tenidos en cuenta en el trabajo con ellos, se relacionan con la gran resistencia al cambio y la terquedad que presentan (Vega, 2001; Vived, 2004) y con su impulsividad (Kopp, Krakow y Johson, 1983) lo que es concordante con los resultados obtenidos en este trabajo. Tambien se ha señalado su orientación emocional que los lleva a requerir mayor regulación externa (Glen y Cunnigham, 2002) y su buena competencia social (Fidler y Nadel, 2007), estos aspectos, son los que podrían esta relacionados con la diferencia que hemos observado entre los Down y los otros niños respecto a su motivación en esta situación de aprendizaje- mediacional que forma parte de la EHPAP.

En definitiva, el trabajo que se presenta arroja luz sobre las diferencias comportamentales entre los niños preescolares Down y los no- Down que deben ser tendidas en cuenta a la hora de programas intervenciones educativas para el primer grupo de niños. Además hemos podido comprobar como niños Down en etapa preescolar presentan una modificabilidad significativa de sus ejecuciones deficitarias y como esa respuesta positiva al entrenamiento se manifiesta no sólo en su ejecución sino también en la conducta que manifiestan durante la sesión de intervención cuando esta sigue un esquema mediacional con un fuerte componente social. Desde nuestro punto de vista, el que estos resultados se hayan comprobado en niños Down preescolares tiene un interés añadido pues nos indican como la intervención etapas inciales de aprendizaje puede tener un efecto significativo sobre todo si atiende a sus déficits específicos. 
Por último, los resultados obtenidos ponen de manifiesto como los instrumentos basados en un esquema dinámico muestran las posibilidades de mejora de los niños con déficits y orientan sobre aspectos no intelectivos muy relevantes a la hora de planificar intervenciones útiles.

La principal limitación de este trabajo ha sido el número de sujetos que componen la muestra que es relativamente pequeño pero que constituye el total de niños Down de la comunidad en que hemos llevado a cabo la investigación. Deberán no obstante realizarse replicaciones del mismo y comparar los perfiles de respuesta a la mediación de los Down respecto de otros tipos de déficits a fin de conseguir la verdadera especificidad de las intervenciones educativas.

Pensamos no obstante que este trabajo muestra que la EHPAP es un instrumento adecuado para su uso en la población preescolar española con Síndrome de Down, tal y como vaticinaban los autores originales de la escala para el caso de sujetos con necesidades educativas especiales en general.

\section{Referencias}

Alony, S. y Kozulin, A. (2007) Dynamic Assessment of receptive language in children with Down Syndrome. International Journal of Speech-language Pathology, 9 (4), 323-331

Arnáiz, P. (1991) Habilidades psicomotoras básicas en el Síndrome Down. En I. Candel y A. Turpin (ED.) Síndrome down: integración escolar y laboral, (118-141). Murcia: Assido.

Aranov, Z. (1999). The reliability and validity of the Application of Cognitive Function Scale (ACFS) Behavior Rating Scale (BORS). Unpublished Master's Thesis. Touro College, New York, NY.

Bensousan, Y. (2002). The Effectiveness of Mediation on Three subtest of the Application of Cognitive Function Scale, a dynamic assessment procedure for young children. Unpublished Master's Thesis, Touro College, New York.

Brooks, N. D. (1997). An exploratory study into the cognitive modifiability of pre-school children using dynamic assessment. Unpublished Master's Thesis: University of Newcastle-Upon-Tyne, Newcastle, United Kingdom. 
Buckley, S., y Sacks, B.I. (2002). An overview of the development of teenagers with Down Syndrome. Portsmouth, UK: The Down syndrome Educational Trust.

Buckley S., y Perera J. (2005). Habla, lenguaje y comunicación en alumnos con Síndrome de Down. En S. Buckley y J. Perera (Ed.) Desarrollo del habla y del lenguaje en alumnos con Síndrome de Down (volumen II) (pp. 20 -34) Madrid: CEPE.

Buckley S., Bird, G., Sacks, B., y Perera, J. (2005). Una introducción para padres y profesores. En Buckley S., Bird G., Sacks B. y Perera J. Vivir con el Síndrome de Down (volumen IV). (pp 39- 56) Madrid: CEPE.

Budoff, M. (1969). Learning Potential: A supplementary procedure for assessing the ability to reason. Seminars in Psychiatry, 1, 278-290.

Caffrey, E. Fuch, D. y Fuch, L.S. (2008) Predictive validity of dynamic assessment: A review. Journal of Special Education, 41, 254-270

Campione, J.C. y Brown, A.L., (1987). Guided learning and transfer: Implications for approaches to assessment. En N. Frederiksen, r. Glaser, A. Lesgold y M. g. Shafto (Eds.), Diagnostic monitoring of skill and Knowledge acquisition, (pp. 45-67) Hillsdale, NJ: Erlbaum.

Candel, I (2005). Elaboración de un Programa de Atención Temprana. Revista Electrónica de Investigación Psicoeducativa, 3 (7), 151-192.

Calero, M.D., (2004). Validez de la Evaluación del Potencial de Aprendizaje. Psicothema, 16 (2) $217-221$

Calero, M.D. y Marquez J. (1998) Psychometric properties of a Learning Potential Tests for reading: The Picture Word Game. European Journal of Psychological Assesmment, 14 (2) $124-133$.

Calero, M.D., Robles, M.A., Márquez, J y de la Osa, P. (2009) EHPAP: Evaluación de habilidades y potencial de aprendizaje en preescolares. Madrid: EOS.

Carlson, J.S. y Wiedl, K.H. (2000). The Validity of Dynamic Assessment. En C.S. Lidz y J.G. Elliott (Eds) Dynamic Assessment: Prevailing models and applications. (p.p.327-347) New York, Elsevier.

Carselimo, G.A., Marotta, L. y Vicari, S. (1997) Long-term memory in mental retardation: evidence for a specific impairment in subjects with Down's syndrome. Neuropsychologia, 35, 71-79.

Chapman, R.S. y Hesketh, L.J (2000) Behavioral Phenotype of individual with Down Syndrome. Mental Retardation and Developmental Disabilities Research Review, 6, 84-95 
Comes, G., Aznar, C., Contijoch, T. y Vives, M. (2001). Enseñanza inicial de la lectura en niños y niñas con Síndrome de Down. Bordón, 53 (1), 21-28.

Comes, G. (2006) Enseñar a leer al alumnado con síndrome Down. Programas de intervención temprana. Malaga: Ediciones Aljibe.

Dunn, M y Dunn, L (1981): Peabody Picture Vocabulary Test-Revised. Circle Pines, MN: American Guidance Services.

Elliot y Fuchs, (1997). The utility of curriculum-based measurement and performance assessment as alternative to traditional intelligence and achievement tests. School Psychology Review, 26, 224-233.

Fernández Ballesteros, R., y Calero, M.D. (2000). The Assessment of Learning Potential: The EPA Instrument. En C. S. Lidz y J. G. Elliot (Eds.), Advances in Cognition and Educational Practice, 6. (pp. 293-324).Amsterdam: JAI

Feuerstein, R., Rand, Y. y Hoffman, M.B,(1979). The dynamic assessment of retarded performers: The Learning Potential Assessment Device: theory and techniques. Baltimore: U.P.P.

Feuerstein R. Feuerstein, R.S. Falik, L.H. y Rand, Y., (2002) The dynamic assessment of Cognitive modifiability. Jerusalem: I. Centre for the enhancement of Learning Potential

Fidler, D.J. y Nadel, L. (2007) Education and children with Down syndrome: Neuroscience, development and intervention. Mental Retardation and Developmental Disabilities Research Reviews, 13, 262-271.

Flórez, J. (1999) Bases neurobiológicas del aprendizaje. Siglo Cero, 30 (183) 9-27

Fuch, D. y Fuch, L.S. (1996) Introduction to Response to intervention: What, why, and how valid is it?. Reading Research Quarterly, 41 (1) 93- 99

Fuchs, L., Fuchs, D., y Hamlett, C. (1994). Strengthening the connection between assessment and instructional planning with expert systems. Exceptional Children, 61 (2), 138-146.

Glen, S. y Cunningham, C. (2002). Self-regulation in children and young people with Down Syndrome. En M. Cuskelly, A. Jobling, y S. Buckley (Eds.). Down syndrome: Across the life span (p.p. 28-39). London: Whurr Publishers.

Gunn D. M., y Jarrold C. (2004). Raven's matrices performance in Down Syndrome: Evidence of unusual errors. Research in Developmental Disabilities 25, 443-457. Berlin: Elsevier.

Haywood, HC., y Lidz, C.S. (2007). Dynamic Assessment in Practice: Clinical and Educational Applications. Cambridge: Cambridge University Press. 
Hessels-Schaltter, C. (2002). Moderate Mental Retardation and Learning Capacity: The Analogical Reasoning Learning Test. En G.M. van de Aslsvoort, W.C. M. Resing y A.J.J.M. Ruijssenaars (Eds.) Learning Potential Assessment and Cognitive Training, 7 (pp. 249-271) Elsevier Science.

Kennedy, M. y Sheridan, C. (1973) Tactile visual equivalence of shape and bland in brain damaged and mongoloid children. Perceptual and Motor Skill, 36 (6) 24-32

Kopp, C. Krakow, J. y Johnson, K. (1983) Strategy production by young Down Syndrome children. American Journal of Mental Deficiency, 88 (2) 193-207

Lauchlan, F., y Elliott, J. (2001). The psychological assessment of learning potential. British Journal of Educational Psychology, 71, 34-66.

Laws, G. y Bishop, D.V. (2003) A comparison of Language Abilities in adolescents with Down syndrome and children with specific language impairment. Journal of Speech, Language and Hearing Research, 46 (6) 1324-1339

Levy, C. (1999). The discriminant validity of the Application of Cognitive Functions Scale (ACFS): A performance comparison between typically developing and special needs preschool children. Unpublished Master's Thesis, Touro College, New York.

Lidz,, C.S. (1991) Practitioner's guide to Dynamic Assessment. N. York: The Guilford Press.

Lidz, C.S. (2002) Mediated Learning Experience (MLE) as a basis form an alternative approach to assessment. School Psychology International, 23 (1) 68-84.

Lidz, C.S. (2004). Assessment procedure with deaf student between the ages of for and eight years. Educational and Child Psychology, 21 (1) 224-240.

Lidz, C.S. y Gindis, B. (2003). Dynamic Assessment of the evolving Cognitive Functions in Children. E A. Kozulin, B. Gindis, V. Ageyev y S. Miller (Eds.). Vygotsky's Educational Theory in Cultural Context (pp. 99-116). London: Cambrigde Unversity Press.

Lidz, C. S. y Jepsen, R. H. (2000). The Application of Cognitive Functions Scale, Administration Manual. New York: Graduate School of Education and Psychology, Touro College.

Lidz, C. S. y Jepsen, R. H. (2003). Application of Cognitive Functions Scale (ACFS). Technical Manual. Manuscrito no publicado.

Lidz, C.S. y Thomas, C. (1987) The Preschool Learning Assessment device: Extension of a static Approach. En C. Lidz (Ed.), Dynamic Assessment: an Interactional Approach to Evaluating Learning Potential (pp. 288-326). New York: Guildford Press. 
Lidz, C.M., y Van der Aalsvoort, G.M. (2005). Usefulness of the Application of Cognitive Functions Scale with Young Children from the Netherlands. Translyvanian Journal of Psychology, 6, 25-44.

Malowitsky, M. (2001). Investigation of the effectiveness of the mediation portion of two subtests of the Application of Cognitive Function Scale, a dynamic assessment procedure for young children. Master's Thesis. Touro College, New York, NY.

Molina S., y Arraiz A. (1993). Procesos y estrategias cognitivas en niños deficientes mentales. Madrid: Ediciones Pirámide.

Molina , S.(2002) Psicopedagogía del niño con síndrome de down. Barcelona: Ariel.

Nadel, L., (2000) Aprendizaje y memoria en síndrome Down. En L. Rondal, L. Perera y L. Nadel (Ed.) Síndrome Down: una revisión de los últimos conocimientos. (p.p.197209). Madrid: Espasa-Calpe

Nihlom, C. (1999) The zone of proximal development: A comparison of children with Down syndrome and typical children. Journal of Intellectual and Developmental Disability, 24 (3) 265-279.

Ortega, J.M. (2002). Nuevas Tecnologías y Aprendizaje Matemático en niños con Síndrome de Down. Ganadora del II Premio en Investigación no médica en Síndrome de Down. Edita: Obra Social de Caja Madrid.

Pérez Sánchez, L. F. Beltrán, J. y Sánchez, E, (2006) Programa de entrenamiento para la mejora de los déficits de memoria en personas con síndrome de Down. Psicothema, 18 (3) $531-536$.

Pino, O. (2000) The effect of context on mother's interaction style with down syndrome and typically developing children. Research in Developmental Disabilities, 21, 329-346

Robles M.A. y Calero M.D. (2003). Evaluación del Potencial de Aprendizaje de la lectura en Síndrome de Down. Siglo Cero Revista Española sobre Discapacidad Intelectual 34 (2)14-26.

Rodríguez, A (2005). Investigación sobre las necesidades educativas especiales: ¿Qué y cómo investigar en la Educación Especial? Electronic Journal of Educational Research in Educational Psychology, 3 (5) 97-112.

Rondal, J.A. (2000) El lenguaje en el retraso mental: diferencias individuales, sindrométricas y variación neurogenética. En Atlas parea volar: la educación como marco para el respeto y la atención a las diferencias. I Congreso I. de N.E.E. Granada: Ed. Adhara (p.p.27-53) 
Ruiz, E. (2001) Evaluación de la capacidad intelectual en personas con Síndrome Down. Revista Síndrome Down, 21, 134-149

Shamir, A. y Lazerowitz (2007) Peer mediation intervention for scaffolding self-regulated learning among children with learning disabilities. European Journal of Special Needs Education, 22 (3) 255-273.

Shurin, R. (1998). Concurrent and discriminant validity assessment procedure with special needs and typical prescool children. Unpublished Master's Thesis. Touro College. New York. NY.

Sternberg, R.J., y Grigorenko E.L. (2003) Evaluación Dinámica. Naturaleza y mediación del potencial de aprendizaje. Madrid, Paidós.

Swanson, H. L. y Lussier, c.M. (2001) A selective synthesis of the experimental literature on Dynamic Assessment. Review of Educational Research, 71 (2) 321-363.

Tarik, T. (2000) A Concurrent validity study between the Application of cognitive Functions Scale and the Leiter-revised International Performance Test. Doctoral dissertation. Touro College, N. York .

Tzuriel, D. y Klein, P.S. (1985). Analogical thinking modifiability in disadvantaged, regular, special education, and mentally retarded children. Journal of Abnormal Child Psychology, 13, 539-552.

Taubman, M. Vrierley, S., Wishner, J., Baker, D., McEachin, J. y Leaf, R.B (2001) The effectiveness of a group discrete trial instructional approach for preschooler with developmental disabilities. Research in Developmental Disabilities, 22, 205-219.

Vega, A. (2001). La educación de los niños con Síndrome de Down. Principios y prácticas. Ediciones Amarú.

Vived, E. (2004). Desarrollo de habilidades cognitivas. En Molina García, S (Dir.) Diseño curricular para alumnos con Síndrome de Down. (pp. 175-212). Edita: Prensas Universitarias de Zaragoza.

Vigotsky, L.S. (1078) El desarrollo de los procesos psicológicos superiores. Barcelona: GRojalbo

William, K.R. Wishart, J.G. Pittcairn, T.K. y Willis, S.S (2005) Emotion recognition by children with Down syndrome: investigation of specifics impairment and error pattern. American Journal of Mental Retardation, 110 (5) 378-392 